\title{
Effects of Neighbourhood Structure on Evolution of Cooperation in N-Player Iterated Prisoner's Dilemma
}

\author{
Raymond Chiong $^{1}$, Sandeep Dhakal ${ }^{1}$ and Lubo Jankovic ${ }^{2}$ \\ ${ }^{1}$ School of IT \& Multimedia, Swinburne University of Technology (Sarawak Campus), \\ Kompleks Negeri, Jalan Simpang Tiga, 93576 Kuching, Sarawak, Malaysia \\ rchiong@swinburne.edu.my, sandeep.dhakal@gmail.com \\ ${ }^{2}$ InteSys Ltd, Birmingham Research Park, Vincent Drive, Birmingham B15 2SQ, UK \\ L.Jankovic@e-intesys.com
}

\begin{abstract}
In multi-agent systems, complex and dynamic interactions often emerge among individual agents. The ability of each agent to learn adaptively is therefore important for them to survive in such changing environment. In this paper, we consider the effects of neighbourhood structure on the evolution of cooperative behaviour in the N-Player Iterated Prisoner's Dilemma (NIPD). We simulate the NIPD as a bidding game on a two dimensional grid-world, where each agent has to bid against its neighbours based on a chosen game strategy. We conduct experiments with three different types of neighbourhood structures, namely the triangular neighbourhood structure, the rectangular neighbourhood structure and the random pairing structure. Our results show that cooperation does emerge under the triangular neighbourhood structure, but defection prevails under the rectangular neighbourhood structure as well as the random pairing structure.
\end{abstract}

Keywords: N-player iterated prisoner's dilemma, bidding game, game strategies, neighbourhood structure.

\section{Introduction}

The Iterated Prisoner's Dilemma (IPD) game has been used extensively to study the evolution of cooperative behaviour over the last two decades [1-4]. However, the game is somewhat limited as it is only between two players. Many real world problems, especially the social and economic ones, often require interactions among various parties. As such, an extension to the IPD game which is more realistic can be found in the multiplayer version game called N-Player IPD (NIPD) [5-6].

NIPD is a collective game that involves more than two players. Each player can either cooperate or defect against other players, getting a reward which increases when there are more players cooperating. This allows the simulations of many aspects of the real world societies, where one can choose to help the population as a whole or help himself at the expense of others.

In this paper, we consider the effects of neighbourhood structure on the evolution of cooperative behaviour in the NIPD game. Previous works have studied the impact of the number of players [6], payoff function [7], neighbourhood size [7], history 
length [8], localisation issue [8], population structure [9], generalisation ability [6, 10], forgiveness [11], trust [12], cultural learning [13], noise [14], etc but none has investigated the influence of the neighbourhood structure on the evolution of cooperation among players in the context of NIPD. We simulate the NIPD as a multiagent bidding game using a two dimensional grid-world, where each agent is required to bid either high or low against its neighbours based on a chosen game strategy. Every agent on the grid-world competes with its neighbours iteratively and gets its scores from their interactions. During the process, the agent looks at its neighbours' scores. If no neighbour has a higher score, the agent retains its original strategy. If the agent has a neighbour or neighbours with higher scores, it will learn to adopt the strategy of the most successful neighbour. With such arrangement, an agent may benefit from actions of other agents whom it interacts with, and it may also take actions that benefit the other agents. The willingness of an agent to take actions which are beneficial to another agent depends strongly on its previous interactions with that agent.

We introduce three different types of neighbourhood structures in which the agents play the bidding game, namely the triangular neighbourhood structure, the rectangular neighbourhood structure and the random pairing structure. We examine these neighbourhood structures with four agents (i.e. $\mathrm{N}=4$ ) playing in a group each time. We expect different neighbourhood structures to affect the outcome of the game differently.

The rest of this paper is organised as follows: Section 2 introduces the background of NIPD. In Section 3, we present the methodology we use for this work. Section 4 describes the experimental setting and results. Finally, we draw conclusion in Section 5 and highlight potential future work.

\section{Background}

The NIPD is an extension of the conventional IPD game in which a group of $\mathrm{N}$ players, where $\mathrm{N}$ is greater than 2 , will continuously interact with each other rather than only two playing against one another. According to Davis et al. [15], NIPD has greater generality and applicability to real life situations than IPD, as many of the real life problems can be represented with the NIPD paradigm in a much realistic way.

In an NIPD game, N players make decision independently on two actions, either cooperate or defect, without knowing other players' choices. Let cooperate be $C$ and defect be $D$, each player attains the score based on the payoff functions $C(\mathrm{c})=\mathrm{c} / \mathrm{N}$ for cooperation and $D(\mathrm{c})=(\mathrm{c}+\mathrm{k}) / \mathrm{N}$ for defection, where $\mathrm{c}$ is the number of cooperators and $\mathrm{k}$ is the total cost for cooperation $(1<\mathrm{k}<\mathrm{N})$ [9]. Players are normally engaged with each other iteratively, competing for higher average scores.

NIPD is also different from the IPD in the kind of strategies that players can adopt successfully. Various strategies that thrive in punishing the defectors in an IPD game, for example Tit For Tat (TFT), a very simple strategy and overall winner of both Axelrod's IPD tournaments [1-2], would not necessarily be so successful in an NIPD game. Punishment handed out to defectors would result in unintentional punishment of other cooperators in the group as well, thus rendering cooperation meaningless to a 
certain extent. This makes the NIPD game more complex and interesting than the IPD game.

\section{Methodology}

The main goal of our work is to examine the effects of neighbourhood structure on the evolution of cooperative behaviour in the NIPD game. In doing so, we construct a two dimensional grid-world in the form of cellular automata to simulate a multi-agent bidding game. Each agent has two actions of either to bid high or to bid low. Every agent will play the bidding game against its neighbours in a group of four (i.e. $N=4$ ), and their actions will be determined by the strategy they choose. The outcome of the game is decided by the payoff shown in Table 1 below.

Table 1. The payoff for our bidding game with $\mathrm{N}=4$.

\begin{tabular}{|c|c|}
\hline Bid Results & Payoff \\
\hline 4 high & -1 \\
\hline 3 high & 1 \\
1 low & -3 \\
\hline 2 high & 3 \\
2 low & -1 \\
\hline 1 high & 5 \\
3 low & 1 \\
\hline 4 low & 3 \\
\hline
\end{tabular}

From Table 1 we see that bidding high is dominant for each agent, because bidding high always yields a better payoff than bidding low no matter how many of the other agents bid low. However, should all agents choose the non-dominant low bids, the outcome would be much better for the group as it yields a total payoff of 12, the highest total payoff among the overall bid results.

\subsection{The Cellular Automata Model}

Our world is implemented in the form of cellular automata on a $20 \times 20$ grid, within a $\mathrm{C}++$ program to simulate agents with different strategies. The grid is formed by a two dimensional array with overlapping edges, which means every cell on the grid has eight immediate neighbouring cells, including those at the edges of the grid. Each agent occupies one cell on the grid, thus there are 400 agents in total in the gridworld. Different colours are used for the cells to represent different strategies adopted by the agents. Each agent is designed to compete against its three immediate neighbours with its initial strategy. Scores are calculated continuously while the game is in progress, and the agents with less successful strategies will start learning the more successful strategies adopted by the neighbouring agents.

We introduced three different neighbourhood structures in our experiments, namely the triangular neighbourhood structure, the rectangular neighbourhood 
structure and the random pairing structure. Figure 1 shows the spatial interactions among agents with the three different types of neighbourhood structures. It is necessary to note that for random pairing structure, the interactions among agents in a group are not confined to the eight immediate neighbours only, but any two agents throughout the grid-world can be paired at random.

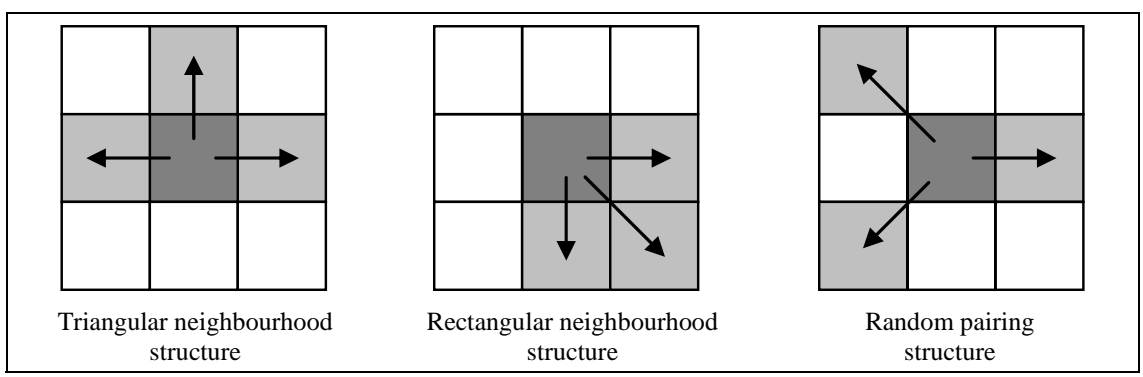

Fig. 1. Three different types of neighbourhood structures.

\subsection{Strategy Representation}

The game strategies that determine the agents' moves can be represented in many different ways. A good representation is always important for this kind of study to ensure the accuracy of results. After much consideration, we have decided to adopt the representation developed by Yao and Darwen [6] as it is exponentially much shorter and easier to implement.

Under this representation, a history of $l$ rounds for an agent can be represented as the combination of the following:

- $\quad l$ bits to represent the agent's $l$ previous bids. Here, a ' 1 ' indicates a high bid, and a ' 0 ' indicates a low bid.

- $\quad l * \log _{2} \mathrm{~N}$ bits to represent the number of low bidders in the previous $l$ rounds among the agent's $\mathrm{N}-1$ group members. Here, $\mathrm{N}$ is the group size.

In this paper, our group size is 4 . We have limited the number of previous bids in memory to 3 (i.e. $l=3$ ). Therefore, based on the above representation scheme, the history for an agent would be $3+3 \log _{2} 4=9$ bits long.

For example, an agent in our bidding game could have a history as follows: 110110110

Here, the first 3 bits are the agent's previous three moves. This means that the agent bid high in the last 2 rounds, and bid low three rounds before. The subsequent 6 bits indicate that all its group members, i.e. $\mathrm{N}-1=3$, bid low in the last round, one group member bid low in the round before last round, and two group members bid low three rounds ago.

Since each history bit string is 9 bits long, there are $2^{9}=512$ possible histories in our game. Due to the fact that there is no memory of previous rounds at the beginning of the game, the agents' history would not be 9 bits long for the first three rounds. They would normally be 3 and 6 bits long at the beginning of the $2^{\text {nd }}$ and $3^{\text {rd }}$ rounds respectively. 
As each strategy we employ in the game needs to have a response for every possible history, each strategy is at least 512 bits long. Depending on different strategies, some strategies would require a maximum of three predefined bids when the history is less than the required 9 bits in length. Hence, our strategies generally range from 512 to 515 bits in length.

\subsection{Strategy Choice}

After devising a way for the strategy representation, an initial population of agents with different strategies needs to be determined. It is impossible for us to include all the game strategies from the entire history of the IPD literature in our simulation experiments, therefore only twelve strategies are being chosen, some of which are distinctive, and some are intelligent. We present these twelve strategies in the $\mathrm{N}$ player versions. For example, an N-player version of TFT will bid low on the first move and then bid whatever the majority of its group members bid in the previous round. Table 2 summarises all the twelve strategies in $\mathrm{N}$-player versions.

We distribute the twelve strategies throughout the grid-world, with each agent acquiring its own initial strategy to play the bidding game. Based on the selection of strategies, every agent starts bidding either high or low during each interaction. Various colours are used to represent different strategies selected by different agents. When an agent learns and adopts another strategy from a more successful agent in order to win the game, the colour of the cell where the agent resides will change, reflecting the new strategy of its choice.

Table 2. The twelve strategies in N-player versions.

\begin{tabular}{|l|l|}
\hline Strategies & \multicolumn{1}{|c|}{ Description } \\
\hline All_High & Bids high all the time. \\
\hline All_Low & Bids low all the time. \\
\hline High_Low & Takes turns to bid high and low. Bids high the first time. \\
\hline Low_High & Takes turns to bid high and low. Bids low the first time. \\
\hline Per_hhl & Bids in the sequence [high, high, low]. \\
\hline Per_llh & Bids in the sequence [low, low, high]. \\
\hline Tit For Tat (TFT) & $\begin{array}{l}\text { Bids low on the first move and then bids what the majority of its } \\
\text { neighbours bid in the previous round. }\end{array}$ \\
\hline Prober & $\begin{array}{l}\text { Begins by bidding [low, high, high]. Continues to bid high if the } \\
\text { majority of the opponents bid low in the previous two rounds. } \\
\text { Otherwise, plays TFT. }\end{array}$ \\
\hline Mistrust & $\begin{array}{l}\text { Bids high on the first move and then bids what the majority of its } \\
\text { neighbours bid in the previous round. }\end{array}$ \\
\hline Pavlov & $\begin{array}{l}\text { Bids high on the first move and then bids low only if the } \\
\text { majority of its neighbours bid the same as itself. }\end{array}$ \\
\hline TF2T & $\begin{array}{l}\text { Bids low from the beginning. Bids high only if the majority of } \\
\text { its neighbours bid high for two consecutive rounds. }\end{array}$ \\
\hline Spiteful & $\begin{array}{l}\text { Bids low until the majority of its neighbours bid high, then bids } \\
\text { high all the time. }\end{array}$ \\
\hline
\end{tabular}




\section{Experiments and Results}

In this section, we present experiments undertaken and discuss the corresponding results based on the twelve strategies described in the previous section. As our objective is to examine the effects of the neighbourhood structure on the evolution of cooperative behaviour in the bidding game, three separate sets of experiments are conducted on the different types of neighbourhood structures. For all our experiments, we use the population size of 400 agents in which all the agents are inhibited on a 20 x 20 grid-world. The twelve strategies selected are randomly distributed among the agents, as evenly as possible, in the beginning of the experiments (see Figure 2). All the experiments are designed for all agents to play against one another iteratively with a group size of four for 200 generations. In every generation, each agent evaluates its neighbours' average scores. If no neighbour has a higher average score, the agent retains its original strategy. If the agent has a neighbour or neighbours with higher average scores, it will learn to adopt the strategy of the most successful neighbour. Scoring in the game is based on the payoff mentioned in Table 1.

It is necessary to highlight here that the average scores are used to evaluate the agents' performance rather than individual scores. This is because individual score only tells us how well an agent is doing as an individual, but average score tells us how well it is doing in its group. Individual scores may be used in an IPD game but are not so suitable for an NIPD game.

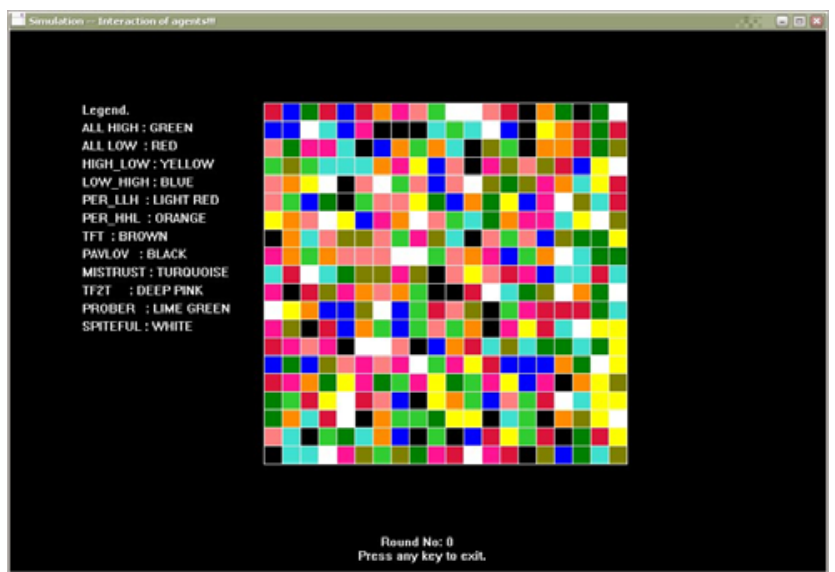

Fig. 2. Initial distribution of the twelve strategies in the beginning of the experiment.

\subsection{Experimental Results on Triangular Neighbourhood Structure}

We have shown earlier in Figure 1 that in the triangular neighbourhood structure, each agent has three group members with whom it interacts, one immediately above itself, one to its left and another to its right. Meanwhile, the agent's group members will also have other different groups associating to them. This allows overlapping between different groups of agents during the game. 
In our first experiment with the triangular neighbourhood structure, we saw that All_Low and strategies that reciprocate against the opponents were dominant. Noncooperative strategies like All_High, Per_hhl, etc disappeared early in the game. This means that the agents are preferring cooperation over defection under this neighbourhood structure. From Figure 3 we observed that even though there were equal or near equal amount of cooperators and defectors in the beginning, almost all the agents were cooperating after 20-30 generations. In most cases, there would be no defectors left at that point.

We believe that the emergence of cooperation via the triangular neighbourhood structure is due to the ability of the agents to reciprocate cooperation and retaliate against defection. Owing to the overlapping nature of the structure, every agent who is interacting with its own group members is also a member of the groups of its neighbours to the left and the right. This means that the agent can indirectly influence the average group scores of two of its neighbours, thus significantly increases the agent's bargaining power against its group members, thereby leading to cooperation.

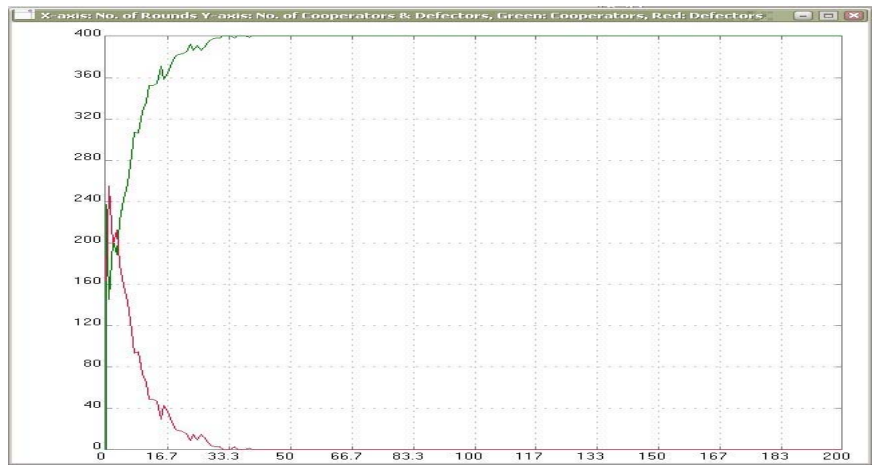

Fig. 3. The number of cooperators (green) vs. defectors (red) in triangular neighbourhood structure.

\subsection{Experimental Results on Rectangular Neighbourhood Structure}

In the case of the rectangular neighbourhood structure, each agent has one group member to its right, one below itself and another one diagonally below it to the right. As with the triangular structure, the group of the agent and the groups of its neighbours are overlapping. However, unlike the triangular structure, the agent itself does not belong to any of the groups of its neighbours.

In our second experiment with the rectangular neighbourhood structure, we noticed that Per_hhl and strategies that tend to follow their opponents did significantly well. Cooperative strategies mostly disappeared early in the game, and even All_High was not doing so well. Although most of the agents were bidding high regularly, they occasionally bid low as well. Only at a few instances was All_High more dominant than other strategies. Even in those instances there was always a strong presence of strategies which would bid low occasionally like Per_hhl, high_low, etc. The graph in Figure 4 evidenced this fact that even though most of the agents were bidding high 
customarily, there were always some agents who were bidding low. In a few cases, the population of cooperators was as high as one fourth of the entire population.

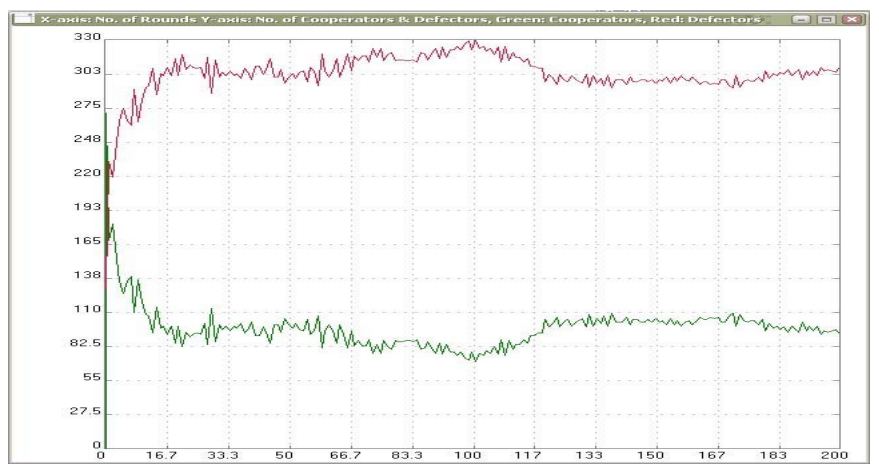

Fig. 4. The number of cooperators (green) $v s$. defectors (red) in rectangular neighbourhood structure.

The result with rectangular neighbourhood structure is rather sluggish as compared to the triangular neighbourhood structure. Nevertheless, defective behaviour is favoured as the agents do not have much chance of retaliating against defectors. This minimises the impact of the significance given to the group rationality, allowing agents to promote their own interests. Still, the emphasis given to group interests in the game ensures that a small percentage of cooperators always existed. The cooperators, though their individual scoring is being hurt by the defectors, would not look to defect because their groups are doing as well as others in most cases. They cannot induce cooperation, though, because they lack the power to retaliate. The defectors, on the other hand, keep on defecting, without any fear of retaliation.

\subsection{Experimental Results on Random Pairing Structure}

In random pairing structure, each agent's group members are selected randomly from the population. The groups would change dynamically after each generation is played. In comparison with the triangular and rectangular structures, where each agent is a member of four overlapping groups, the random pairing allows an agent to be selected in a maximum of four groups. Due to the random pairing nature, the chances that there is overlap between the agent's group and the groups of its group members are minimal.

In our third experiment with the random pairing structure, we observed that defection was rampant among the population from very early in the game. The grid would normally show no changes in the agents' strategies after about 20-30 generations. All the cooperative strategies vanished before they could reach the $30^{\text {th }}$ generation. All_High was quite dominant along with the likes of TFT, Mistrust, Spiteful, etc. All the latter strategies were second best to All_High in no particular order. The strength of their performance was also arbitrary. Figure 5 shows the result where, by around 30 generations onwards, there were literally no more cooperators 
left in the game. Since all the agents were defecting and getting similar scores, there was no need for a strategy change.

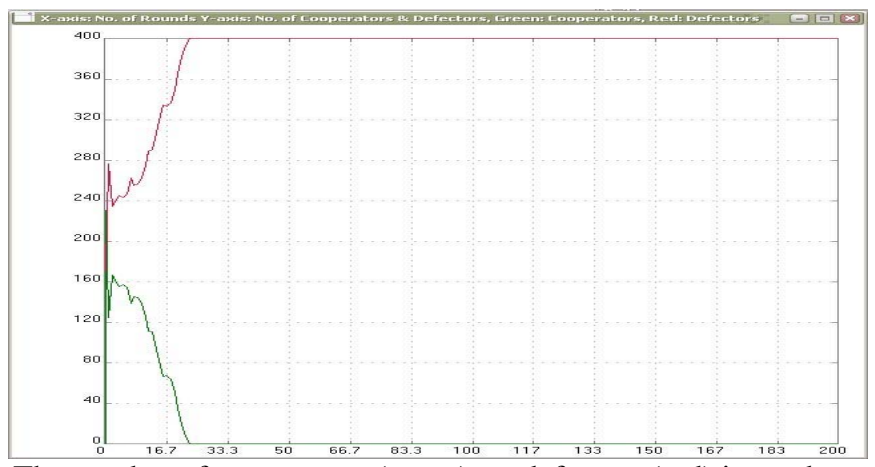

Fig. 5. The number of cooperators (green) vs. defectors (red) in random pairing structure.

It is easy to see why defection is so dominant in the case of random pairing. Due to the randomly pitted interactions, anonymity is often guaranteed as the chances of the same group members meeting one another again are extremely small. As there is no fear of reciprocation or retaliation, there is little pressure for cooperative behaviour. This result concurs with Ishibuchi and Namikawa [16] who used a similar random pairing scheme in their studies on evolving IPD game strategies under structured demes. They demonstrated that the evolution of reciprocal strategies is very difficult to achieve when opponents are selected randomly.

\section{Conclusion}

In this paper, we investigated the effects of three different neighbourhood structures on the emergence of cooperation in a multi-agent bidding game. Our experimental results demonstrated that agents need to have the ability to punish defection in order to induce cooperation from a population with numerous defectors. This occurs in overlapping neighbourhoods in which one agent is at a center of one neighbourhood, and at a fringe of another. In this way the behaviour of each agent influences more than one neighbourhood, and results in higher memory of the system and higher cooperation. That is why cooperation emerges in the triangular neighbourhood structure but not in the rectangular and the random pairing neighbourhood structures. We also showed that without the fear of being retaliated against, defectors and players with opportunistic strategies would make use of the cooperators around them only to forward their self-interests.

In our current work the agents are limited to choosing only from a fixed set of strategies and they lack the ability to alter their strategies. However, alteration rather than complete abandonment of their strategies would be closer to the rules of nature and reality. Therefore, the future work will involve the use of co-evolutionary 
learning within the population. This would enable us to see whether or not coevolution can induce the emergence of cooperation in less favourable neighbourhood structures such as the rectangular or random pairing.

\section{References}

1. Axelrod, R.: The Evolution of Cooperation. Basic Books, New York (1984)

2. Axelrod, R.: The Evolution of Strategies in the Iterated Prisoner's Dilemma. In: Davis, L. (ed.): Genetic Algorithms and Simulated Annealing, pp. 32--41. Morgan Kaufmann, Los Altos, CA (1987)

3. Lindgren, K.: Evolution Phenomena in Simple Dynamics. In: Langton, C.G., Taylor, C., Farmer, J.D., Rasmussen, S. (eds.): Artificial Life II, pp. 295--312. Addison-Wesley, Reading, MA (1991)

4. Fogel, D.B.: Evolving Behaviors in the Iterated Prisoner's Dilemma. Evolutionary Computation. 1(1), 77--97 (1993)

5. Bankes, S.: Exploring the Foundations of Artificial Societies: Experiments in Evolving Solutions to Iterated N-player Prisoner's Dilemma. In: Brooks R.A., Maes, P. (eds.): Artificial Life IV, pp. 237--242. MIT Press, Cambridge, MA (1994)

6. Yao, X., Darwen, P.: An Experimental Study of N-person Iterated Prisoner's Dilemma Games. Informatica. 18(4), 435--450 (1994)

7. Seo, Y.G., Cho, S.B., Yao, X.: The Impact of Payoff Function and Local Interaction on the N-player Iterated Prisoner's Dilemma. Knowledge and Information Systems: An International Journal. 2(4), 461--178 (2000)

8. Seo, Y.G., Cho, S.B., Yao, X.: Emergence of Cooperative Coalition in NIPD Game with Localization of Interaction and Learning. In: Proceedings of the 1999 Congress on Evolutionary Computation, pp. 877--884. IEEE Press, Piscataway, NJ (1999)

9. Suzuki, R., Arita, T.: Evolutionary Analysis on Spatial Locality in N-Person Iterated Prisoner's Dilemma. International Journal of Computational Intelligence and Applications. 3(2), 177--188 (2003)

10. Yao, X.: Automatic Acquisition of Strategies by Co-evolutionary Learning. In: Proceedings of the International Conference on Computational Intelligence and Multimedia Applications, Gold Coast, Australia, pp. 23--29. Griffith University Press, Brisbane, Australia (1997)

11. O’Riordan, C., Griffith, J., Newell, J., Sorensen, H.: Co-evolution of Strategies for an Nplayer Dilemma. In: Proceedings of the 2004 Congress on Evolutionary Computation, pp. 1625--1630. IEEE Press, Piscataway, NJ (2004)

12. Birk, A.: Trust in an N-player Iterated Prisoner's Dilemma. In: Proceedings of the International Conference on Autonomous Agents, $2^{\text {nd }}$ Workshop on Deception, Fraud and Trust in Agent Societies, Seattle, WA (1999)

13. O’Riordan, C., Griffith, J., Curran, D., Sorensen, H.: Norms and Cultural Learning in the Nplayer Prisoner's Dilemma. In: Proceedings of the 2006 Congress on Evolutionary Computation, pp. 1105--1110. IEEE Press, Piscataway, NJ (2006)

14. Chong, S.Y., Yao, X.: Behavioral Diversity, Choices and Noise in the Iterated Prisoner's Dilemma. IEEE Transactions on Evolutionary Computation. 9(6), 540--551 (2005)

15. Davis, J.H., Laughlin, P.R., Komorita, S.S.: The Social Psychology of Small Groups. Annual Review of Psychology. 27, 501--542 (1976)

16. Ishibuchi, H., Namikawa, N.: Evolution of Iterated Prisoner's Dilemma Game Strategies in Structured Demes under Random Pairing in Game Playing. IEEE Transactions on Evolutionary Computation. 9(6), 552--561 (2005) 